[18] P. A. Anghel and M. Kaveh, "Exact symbol error probability of a cooperative network in a Rayleigh-fading environment," IEEE Trans. Wireless Commun., vol. 3, no. 5, pp. 1416-1421, Sep. 2004.

[19] S. Ikki and M. H. Ahmed, "Performance analysis of cooperative diversity wireless networks over Nakagami- $m$ fading channel," IEEE Commun. Lett., vol. 11, no. 4, pp. 334-336, Apr. 2007.

[20] T. Nechiporenko, P. Kalansuriya, and C. Tellambura, "Performance of optimum switching adaptive $M$-QAM for amplify-and-forward relays," IEEE Trans. Veh. Technol., vol. 58, no. 5, pp. 2258-2268, Jun. 2009

\section{Distributed Self-Concatenated Coding for Cooperative Communication}

Muhammad Fasih Uddin Butt, Student Member, IEEE, Raja Ali Riaz, Soon Xin Ng, Senior Member, IEEE, and Lajos Hanzo, Fellow, IEEE

\begin{abstract}
In this paper, we propose a power-efficient distributed binary self-concatenated coding scheme using iterative decoding (DSECCCID) for cooperative communications. The DSECCC-ID scheme is designed with the aid of binary extrinsic information transfer (EXIT) charts. The source node transmits self-concatenated convolutional coded (SECCC) symbols to both the relay and destination nodes during the first transmission period. The relay performs SECCC-ID decoding, where it may or may not encounter decoding errors. It then reencodes the information bits using a recursive systematic convolutional (RSC) code during the second transmission period. The resultant symbols transmitted from the source and relay nodes can be viewed as the coded symbols of a three-component parallel concatenated encoder. At the destination node, three-component DSECCC-ID decoding is performed. The EXIT chart gives us an insight into operation of the distributed coding scheme, which enables us to significantly reduce the transmit power by about $3.3 \mathrm{~dB}$ in signal-to-noise ratio (SNR) terms, as compared with a noncooperative SECCC-ID scheme at a bit error rate (BER) of $10^{-5}$. Finally, the proposed system is capable of performing within about $1.5 \mathrm{~dB}$ from the two-hop relay-aided network's capacity at a BER of $10^{-5}$, even if there may be decoding errors at the relay.
\end{abstract}

Index Terms-Cooperation diversity, distributed coding, extrinsic information transfer (EXIT) charts, iterative decoding, self-concatenated coding.

\section{INTRODUCTION}

Digital communication exploiting multiple-input-multiple-output (MIMO) wireless channels have recently attracted considerable attention. The wireless communication systems of future generations are required to provide reliable transmissions at high data rates to offer a variety of multimedia services to commercial wireless products and

Manuscript received July 14, 2009; revised April 13, 2010; accepted April 15, 2010. Date of publication April 29, 2010; date of current version July 16, 2010. This work was supported by the COMSATS Institute of Information Technology under the auspices of Higher Education Commission, Pakistan, and that of the Engineering and Physical Sciences Research Council, U.K., as well as of the European Union Optimix Project. The review of this paper was coordinated by M. Dohler.

M. F. U. Butt and R. A. Riaz are with the School of Electronics and Computer Science, University of Southampton, Southampton SO17 1BJ, U.K., and also with the Department of Electrical Engineering, COMSATS Institute of Information Technology, Islamabad 44000, Pakistan (e-mail: mfub06r@ecs.soton.ac.uk; rar06r@ecs.soton.ac.uk).

S. X. Ng and L. Hanzo are with the School of Electronics and Computer Science, University of Southampton, Southampton SO17 1BJ, U.K. (e-mail: sxn@ecs.soton.ac.uk; 1h@ecs.soton.ac.uk).

Color versions of one or more of the figures in this paper are available online at http://ieeexplore.ieee.org.

Digital Object Identifier 10.1109/TVT.2010.2048933 networks. Space-time coding schemes [1], which employ multiple transmitters and receivers, are among the most efficient techniques designed to achieve a high diversity gain, provided that the associated MIMO channels [2], [3] experience independent fading. Utilizing cooperative techniques eliminates the correlation of the signals when using multiple antennas at the mobiles, which is imposed by the limited affordable element spacing.

Although full-duplex relaying and the associated capacity theorem derived for the discrete memoryless relay channel model have been proposed in [4], practical cooperative diversity schemes were only proposed much later in [5]-[8]. In practice, each mobile collaborates with a single or a few partners for the sake of reliably transmitting both its own information and that of its partners jointly, which emulates a virtual MIMO scheme. The most popular collaborative protocols used between the source, relay, and destination nodes are amplify-and-forward, demodulate-and-forward, and decode-andforward (DAF) schemes [9]. We derive the theoretical lower and upper bounds on the continuous-input-continuous-output memoryless channel (CCMC) capacity, as well as of the discrete-input-continuousoutput memoryless channel (DCMC) [3], [4], [10], [11] capacity (constrained information rate) for independent and uniformly distributed (i.u.d.) sources.

The philosophy of concatenated coding schemes was proposed by Forney in [12]. Turbo codes, which were developed in [13], constitute a class of error correction codes based on two or more parallel concatenated convolutional codes that are used as constituent codes. They are high-performance codes capable of operating near the Shannon limit. Since their invention, they have found diverse applications in bandwidth-limited communication systems, where the maximum achievable information rate has to be supported in the presence of transmission errors due to both the ubiquitous additive white Gaussian noise (AWGN) and channel fading. Various bandwidth-efficient turbo codes were proposed in [14]-[16]. Serially concatenated convolutional codes [17] have been shown to yield a performance comparable, and in some cases superior, with turbo codes. Iteratively decoded self-concatenated convolutional codes (SECCC-ID) proposed by Benedetto et al. [18] constitute another attractive family of iterative-detection-aided schemes. The SECCC arrangement is a low-complexity scheme using a single encoder and a single decoder. The extrinsic information transfer (EXIT) chart [19]based analysis of the iterative decoder provides an insight into its decoding convergence behavior, and hence, it is helpful for finding the best constituent coding schemes for creating near-capacity SECCCs both for AWGN and Rayleigh fading channels [20]. EXIT charts constitute a semianalytical tool used to predict the SNR value, where an infinitesimally low bit error ratio (BER) can be achieved without performing time-consuming bit-by-bit decoding, which employs a high number of decoding iterations.

Distributed coding [21] constitutes another attractive cooperative diversity technique, where joint signal design and coding are invoked at the source and relay nodes. Distributed turbo codes [22], [23] have also been proposed for cooperative communications, although typically under the simplifying assumption of having a perfect communication link between the source and relay nodes. These are halfduplex relay-aided systems, where the source transmits to both the relay and the destination during the first transmission period, and after decoding the information from the source, the relay reencodes it and sends it to the destination in the second transmission period. Hence, half-duplex systems do not suffer from multiaccess interference, which results in a simplified receiver structure at the cost of halving the spectral efficiency. As a more realistic design alternative, a 
turbo-coded cooperation-aided system having an imperfect sourcerelay (SR) communication link has been proposed in [24] and [25]. In [24], the source node continues its transmission of the rest of the codewords in the second transmission period with the aim of achieving improved bandwidth efficiency. Still referring to [24], the signals arriving from the source and the relay are superimposed at the destination, where a maximum a posteriori probability (MAP) detector and a turbo decoder exchange extrinsic information, which were shown to be capable of operating near the capacity of ergodic flat-fading channels. The scheme proposed in [25] considers a more complex irregular lowdensity parity-check-coded near-capacity system designed using EXIT charts and a design procedure similar to that of [24]. It is demonstrated in [26] and [27] that, in the presence of Rayleigh fading, DAF cooperation-assisted systems are expected to outperform their noncooperative counterparts. However, an error floor is observed in [26], which can be mitigated by using soft relaying [28], [29]. In this paper, we propose a power-efficient distributed self-concatenated coding scheme using iterative decoding (DSECCC-ID) for cooperative communications. The benefits of our novel solution are given in the list that follows.

1) In our proposed half-duplex relaying system, the sourcedestination (SD) link employs an SECCC code, whereas the relay node employs a simple recursive systematic convolutional (RSC) encoder instead of an SECCC encoder. Therefore, iterative decoding at the destination exchanges information between the SECCC MAP decoder and an RSC MAP decoder.

2) The source employs an SECCC encoder, which reuses the same component instead of having two separate constituent codes. First, SECCC iterative decoding is employed at the relay, which then reencodes the decoded symbols by a low-complexity RSC encoder.

3) The relay frame is shorter than the source frame because of the puncturing of the systematic bits. Hence, the overall throughput is higher.

4) The motivation for using the proposed three-stage decoder architecture is to effectively reduce the error floor documented in [26, Figs. 15.6-15.10] for the conventional two-stage architecture [26, Fig. 15.3]. ${ }^{1}$

5) The proposed scheme is designed by a systematic and widely applicable procedure using EXIT charts.

6) The SR link is imperfect; however, this simplified scheme is capable of approaching capacity.

This paper is organized as follows: The system model is described in Section II. The encoder for DSECCC and its corresponding decoder are highlighted in Sections III and IV, respectively. The design and analysis of the proposed scheme is provided in Section V. Our simulation results are discussed in Section VI. Finally, our conclusions are offered in Section VII.

\section{SYSTEM MODEL}

The schematic of a two-hop, half-duplex, relay-aided system is shown in Fig. 1, where the source node $s$ transmits a frame of coded symbols $\mathbf{x}_{s}$ to both the relay node $r$ and the destination node $d$ during the first transmission period $T_{1}$, whereas the relay node first decodes the information, then reencodes it, and finally transmits a frame of coded symbols $\mathbf{x}_{r}$ to the destination node during the second transmission period $T_{2}$. In the time-division multiple-access protocol used, the source transmits to both the relay and the destination during

\footnotetext{
${ }^{1}$ The authors gratefully acknowledge the Editor's and the reviewers' suggestions to explicitly clarify the motivation for using the proposed architecture.
}

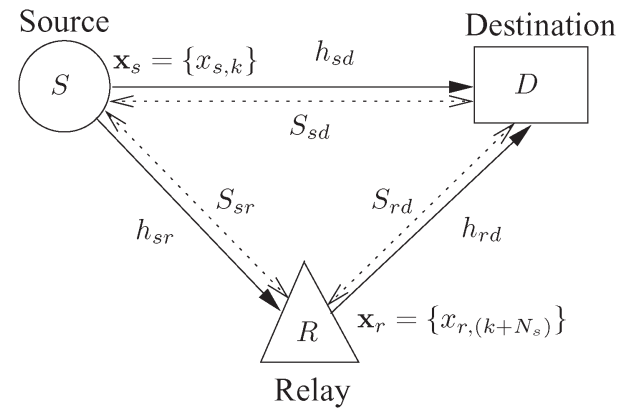

Fig. 1. Schematic of a two-hop relay-aided system, where $S_{a b}$ is the geographical distance between nodes $a$ and $b$.

$T_{1}$, whereas in $T_{2}$, only the relay transmits to the destination. Hence, this protocol was termed as exhibiting degree- 2 of broadcasting and no receive collision in [30]. The communication links shown in Fig. 1 are subject to both free-space path loss and uncorrelated Rayleigh fading.

Let $S_{a b}$ denote the geometrical distance between nodes $a$ and $b$. The path loss between these nodes can be modeled by [8], [31]

$$
P(a b)=K / S_{a b}^{\alpha}
$$

where $K$ is a constant that depends on the environment, and $\alpha$ is the pathloss exponent. For a free-space pathloss model, we have $\alpha=2$. The relationship between the energy $E_{s r}$ received at the relay node and that of the destination node $E_{s d}$ can be expressed as

$$
E_{s r}=\frac{P(s r)}{P(s d)} E_{s d}=G_{s r} E_{s d}
$$

where $G_{s r}$ is the power gain (or geometrical gain) [8] experienced by the SR link with respect to the SD link as a benefit of its reduced distance and path loss, which can be computed as

$$
G_{s r}=\left(\frac{S_{s d}}{S_{s r}}\right)^{2}
$$

Similarly, the power gain for the RD link with respect to the SD link can be formulated as

$$
G_{r d}=\left(\frac{S_{s d}}{S_{r d}}\right)^{2} .
$$

Naturally, the power gain of the SD link with respect to itself is unity, i.e., $G_{s d}=1$.

The $k$ th received signal at the relay node during the first transmission period $T_{1}$, where $N_{s}$ number of symbols are transmitted from the source node, can be written as

$$
y_{r, k}^{\left(T_{1}\right)}=\sqrt{G_{s r}} h_{s r, k}^{\left(T_{1}\right)} x_{s, k}+n_{r, k}^{\left(T_{1}\right)}
$$

where $k \in\left\{1, \ldots, N_{s}\right\}$, and $h_{s r, k}^{\left(T_{1}\right)}$ is the complex-valued Rayleigh fading channel coefficient between the source and the relay at instant $k$, whereas $n_{r, k}^{\left(T_{1}\right)}$ is the zero-mean complex AWGN having a variance of $N_{0} / 2$ per dimension. By contrast, the $k$ th symbol received at the destination during the period $T_{1}$ can be expressed as

$$
y_{d, k}^{\left(T_{1}\right)}=\sqrt{G_{s d}} h_{s d, k}^{\left(T_{1}\right)} x_{s, k}+n_{d, k}^{\left(T_{1}\right)}
$$

where $h_{s d, k}^{\left(T_{1}\right)}$ is the complex-valued Rayleigh fading channel coefficient between the source and the destination at instant $k$, whereas $n_{d, k}^{\left(T_{1}\right)}$ is the AWGN having a variance of $N_{0} / 2$ per dimension. Similarly, the $l$ th symbol received at the destination during the second period $T_{2}$, 


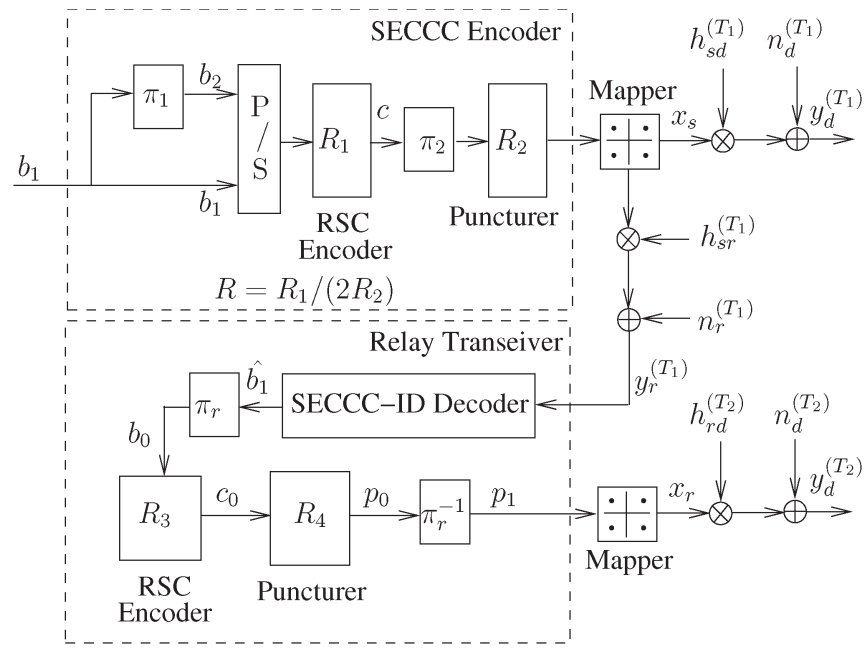

Fig. 2. Schematic of a three-component self-concatenated encoder. This figure applies to the DSECCC-ID scheme when the relay transceiver node decodes the received symbols using the SECCC-ID decoder and then forwards the decoded symbols to the destination in the second phase.

where $N_{r}$ number of symbols are transmitted from the relay node, is given by

$$
y_{d, l}^{\left(T_{2}\right)}=\sqrt{G_{r d}} h_{r d, l}^{\left(T_{2}\right)} x_{r, l}+n_{d, l}^{\left(T_{2}\right)}
$$

where $l \in\left\{1+N_{s}, \ldots, N_{r}+N_{s}\right\}$, and $h_{r d, l}^{\left(T_{2}\right)}$ is the complex-valued Rayleigh fading channel coefficient between the relay and the destination at instant $l$, whereas $n_{d, l}^{\left(T_{2}\right)}$ is the AWGN having a variance of $N_{0} / 2$ per dimension.

\section{Distributed Self-Concatenated Coding EnCoder}

In our DSECCC-ID scheme, we consider a quadrature phase-shift keying (QPSK)-assisted SECCC encoder at the source and a QPSKassisted RSC encoder at the relay.

Note that the relay transmits only the parity bits during the second transmission period to ensure that the systematic bits are transmitted only once to the destination. The relay detects the signals received from the source node during the first transmission period. The notation $\pi_{r}$ in Fig. 2 denotes the random bit interleaver used at the relay to interleave the decoded bits before the RSC encoding. The encoders employed at both the source and relay transceiver nodes can be viewed as a three-component parallel concatenated SECCC encoder, ${ }^{2}$ which is depicted in Fig. 2. The notation $x_{r}$ used in Fig. 2 denotes the 2-bit QPSK symbol at the relay node. The puncturer, which is denoted $R_{4}$ in Fig. 2, is used to improve the overall throughput of the scheme. We found that a good performance can be achieved by transmitting only the parity bits generated at the output of the RSC encoder at the relay node.

At the source node, we consider a rate of $R=1 / 3$ SECCC scheme employing QPSK modulation. Uncorrelated Rayleigh fading channel conditions are considered for this analysis.

As shown in Fig. 2, the input bit sequence $\left\{b_{1}\right\}$ of the selfconcatenated encoder is interleaved for yielding the bit sequence $\left\{b_{2}\right\}$. The resultant bit sequences are parallel-to-serial converted and then fed to the RSC encoder, which employs the generator polynomial $G=$ [13 15 ] expressed in octal format and having a rate of $R_{1}=1 / 2$ and

${ }^{2} \mathrm{An}$ SECCC encoder can be viewed as a two-component parallel concatenated encoder [32]. $\nu=3$. Hence, for every bit input to the SECCC encoder, there are four output bits of the RSC encoder. At the output of the encoder, there is an interleaver and then a rate of $R_{2}=3 / 4$ puncturer, which punctures (does not transmit) one bit out of four encoded bits. Hence, the overall code rate $R$ can be derived based on [33] as

$$
R=\frac{R_{1}}{2 \times R_{2}}=\frac{1 / 2}{2(3 / 4)}=\frac{1}{3} .
$$

Puncturing is used to increase the achievable bandwidth efficiency $\eta$. Different codes have been designed in [20] by changing the rates of $R_{1}$ and $R_{2}$. These bits are then mapped to a QPSK symbol as $x=$ $\mu\left(c_{1} c_{0}\right)$, where $\mu($.$) is the bit-to-symbol mapping function. Hence, the$ bandwidth efficiency is given by $\eta=R \times \log _{2}(4)=0.67 \mathrm{bit} / \mathrm{s} / \mathrm{Hz}$, assuming a zero Nyquist rolloff factor. The QPSK symbol $x_{s}$ is then transmitted over the channel.

The overall throughput of this two-hop cooperative scheme can be formulated as

$$
\eta=\frac{N_{i}}{N_{s}+N_{r}} \quad(\text { in bits/s })
$$

where $N_{i}$ is the number of information bits transmitted within a duration of $\left(N_{s}+N_{r}\right)$ symbol periods. Again, $N_{s}$ is the number of modulated symbols per frame transmitted from the source node, and $N_{r}$ is the number of modulated symbols per frame arriving from the relay node. For our case, we have $N_{i}=120000$ bits. Therefore, we transmit $N_{s}=180000$ symbols. Note that the number of symbols per transmission burst at the relay node is given by $N_{r}=60000$ due to the employment of QPSK modulation and a rate of $R_{4}=1 / 2$ puncturer that removes all systematic bits from the output of the RSC encoder of a rate of $R_{3}=1 / 2$. Hence, the overall effective throughput of the DSECCC-ID scheme is given by $\eta=\left(N_{i}\right) /\left(N_{s}+N_{r}\right)=0.5 \mathrm{bit} / \mathrm{s}$. The SNR per bit is given by $E_{b} / N_{0}=\mathrm{SNR} / \eta$. Hence, the DSECCCID scheme suffers from a penalty of $1.25 \mathrm{~dB}$ in terms of $E_{b} / N_{0}$, as compared with the conventional SECCC-ID scheme having a somewhat higher throughput of $0.67 \mathrm{bit} / \mathrm{s} / \mathrm{Hz}$.

\section{Distributed Self-Concatenated Coding Using ITERATIVE DECODING DECODER}

The novel decoder structure of the DSECCC-ID scheme is illustrated in Fig. 3. The notations $P($.$) and L($.$) in Fig. 3$ denote the logarithmic-domain symbol probabilities and the logarithmiclikelihood ratio (LLR) of the bit probabilities, respectively. The notations $b$ and $c$ in the round brackets (.) in Fig. 3 denote information bits and coded bits, respectively. The specific nature of the probabilities and LLRs is represented by the subscripts $a, o$, and $e$, which denote a priori, a posteriori, and extrinsic information, respectively, in Fig. 3.

For the SECCC-ID decoder, which is denoted by (1) in Fig. 3, the received signal arrives at the soft demapper. This signal is then used by the demapper to calculate the conditional probability density function (pdf) of receiving $\mathbf{y}_{d}^{\left(T_{1}\right)}$ when $\mathbf{x}_{s}$ was transmitted, yielding

$$
p\left(\mathbf{y}_{d}^{\left(T_{1}\right)} \mid \mathbf{x}=\mathbf{x}_{s}^{m}\right)=\frac{1}{\pi N_{0}} \exp \left(-\frac{\left|\mathbf{y}_{d}^{\left(T_{1}\right)}-\mathbf{h}_{s d}^{\left(T_{1}\right)} \mathbf{x}_{s}^{m}\right|^{2}}{N_{0}}\right)
$$

where $\mathbf{x}_{s}^{m}=\mu\left(c_{1} c_{0}\right)$ is the hypothetically transmitted QPSK symbol for $m \in\{0,1,2,3\}, \mathbf{h}_{s d}$ is the channel's nondispersive fading coefficient, and $\mathbf{n}_{d}$ is the AWGN having a variance of $N_{0} / 2$ per dimension. The received signal that arrives at the soft demapper of the SECCC-ID 


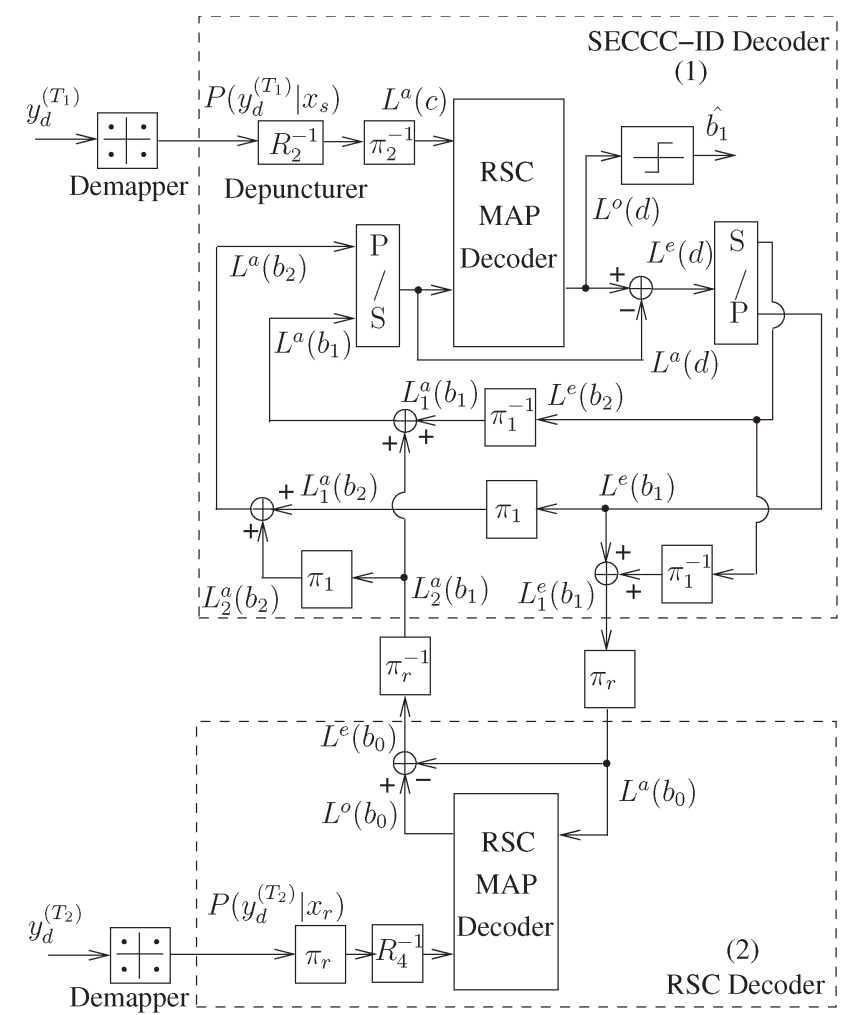

Fig. 3. Schematic of the DSECCC-ID decoder. The input to the SECCC-ID decoder is through the QPSK demapper for an SD link, whereas the input to the RSC decoder is through the QPSK demapper for an RD link.

decoder (1) is given in (10), whereas the received signal that arrives at the soft demapper of the RSC decoder, which is denoted by (2) in Fig. 3, is used to calculate the conditional pdf of receiving $\mathbf{y}_{d}^{\left(T_{2}\right)}$ when $\mathbf{x}_{r}$ was transmitted, yielding

$$
p\left(\mathbf{y}_{d}^{\left(T_{2}\right)} \mid \mathbf{x}=\mathbf{x}_{r}^{m}\right)=\frac{1}{\pi N_{0}} \exp \left(-\frac{\left|\mathbf{y}_{d}^{\left(T_{2}\right)}-\mathbf{h}_{r d}^{\left(T_{2}\right)} \mathbf{x}_{r}^{m}\right|^{2}}{N_{0}}\right)
$$

The extrinsic bit probabilities are then passed through a soft depuncturer, which converts them to the corresponding bit-based LLRs and subsequently inserts zero LLRs at the punctured bit positions. The LLRs are then deinterleaved and fed to the soft-input-soft-output MAP decoder [34]. The decoder of Fig. 3 is a self-concatenated decoder, which can be viewed as a three-component parallel concatenated decoder, which first calculates the extrinsic LLRs of the information bits, namely, $L^{e}\left(b_{1}\right)$ and $L^{e}\left(b_{2}\right)$. Then, they are appropriately interleaved to yield the a priori LLRs of the information bits, namely, $L^{a}\left(b_{1}\right)$ and $L^{a}\left(b_{2}\right)$, as shown in Fig. 3. Self-concatenated decoding proceeds until a fixed number of iterations are reached.

There are two inputs to the RSC MAP decoder block, which is denoted by (2) in Fig. 3. The first is the extrinsic information of bit $b_{1}$ provided by the SECCC-ID decoder, which is denoted by (1). As shown in Fig. 3, this is obtained from the addition of $L^{e}\left(b_{1}\right)$ and the deinterleaved version of $L^{e}\left(b_{2}\right)$. The resultant $L_{1}^{e}\left(b_{1}\right)$ stream is interleaved by $\pi_{r}$ to generate $L^{a}\left(b_{0}\right)$. The second input of the RSC MAP decoder (2) is the interleaved and depunctured version of the soft information provided by the QPSK demapper, which is denoted by $P\left(y_{d}^{T_{2}} \mid x_{r}\right)$ in Fig. 3 . The RSC decoder of the relay shown in Fig. 3 then provides the improved extrinsic LLR of the data bit $b_{0}$, namely, $L^{e}\left(b_{0}\right)$ as its output, which is deinterleaved by $\pi_{r}^{-1}$ to yield $L_{2}^{a}\left(b_{1}\right)$.

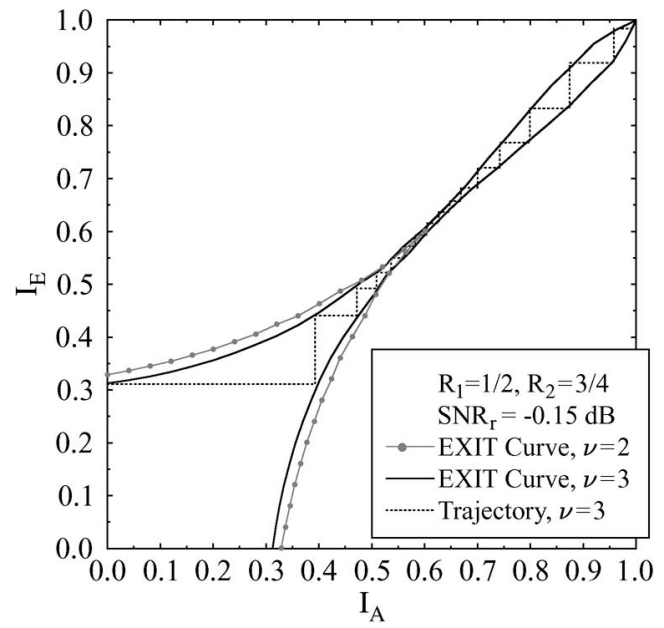

Fig. 4. EXIT curves for $\nu=2$ and $\nu=3, R_{1}=1 / 2$ and $R_{2}=3 / 4$, QPSKassisted SECCC-ID, and $\eta=0.67 \mathrm{bit} / \mathrm{s} / \mathrm{Hz}$ at SNR $r=-0.15 \mathrm{~dB}$ for transmission over an uncorrelated Rayleigh channel. A decoding trajectory for $\nu=3$ is also depicted.

The LLR $L_{2}^{a}\left(b_{1}\right)$ can further be interleaved using $\pi_{1}$ to generate $L_{2}^{a}\left(b_{2}\right)$. These a priori LLR outputs by the RSC can be added to the SECCC-ID decoder's a priori LLRs of $b_{1}$ and $b_{2}$, thus completing the iteration between the RSC and SECCC-ID decoders.

\section{Design And Analysis}

We design and analyze the proposed scheme using EXIT charts in Section V-A. Furthermore, the relay-aided system capacity is detailed in Section V-B.

\section{A. EXIT Charts Analysis}

Binary EXIT charts are useful for finding the best SECCC-ID schemes for having a decoding convergence at the lowest possible SNR value. The EXIT curves of the SECCC decoder components and a corresponding decoding trajectory were recorded for the best performing binary SECCC schemes operating closest to the Rayleigh fading channel's capacity [20]. Since, in the case of SECCCs, there are identical components, we only have to compute the EXIT curve of a single component, and the other is its mirror image [20]. The EXIT curves of the two hypothetical decoder components are plotted within the same EXIT chart together with their corresponding decoding trajectory for the sake of visualizing the exchange of extrinsic information between the decoders. These were recorded by using ten transmission frames, each consisting of 24000 information bits for calculating the EXIT curve, whereas we consider a frame size of 120000 information bits for calculating the decoding trajectories. ${ }^{3}$

Our three-step design procedure using EXIT charts developed for the proposed distributed coded system is given as follows.

Step 1: Our code design procedure commences by calculating the decoding convergence of the SECCC-ID scheme at the output of the communication link between the SR link, using EXIT charts. The bestperforming QPSK-assisted SECCC-ID scheme employs $R_{1}=1 / 2$ and $R_{2}=3 / 4$ having $\eta=0.67 \mathrm{bit} / \mathrm{s} / \mathrm{Hz}$.

As shown in Fig. 4, we compare the SECCC scheme using $\nu=2$ and $\nu=3$ at a receive SNR of about $-0.15 \mathrm{~dB}$. For the

\footnotetext{
${ }^{3}$ We need large interleaver sizes for the trajectories to match the EXIT curves, whereas for the EXIT curves, fewer bits can give us a good prediction [19].
} 
$\nu=3$-SECCC code, a receive SNR of about $-0.15 \mathrm{~dB}$ is needed to attain a decoding convergence to the $(1,1)$ point of the EXIT chart, since at a receive SNR of $-0.2 \mathrm{~dB}$, the EXIT tunnel remains closed. By contrast, the EXIT tunnel for the $\nu=2$ SECCC code remains closed at $-0.15 \mathrm{~dB}$. Consequently, we opted for $\nu=3$, as it requires reduced transmission power. Fig. 4 also corresponds to the performance of the SECCC-ID scheme of the SR link. The receive SNR can be computed as

$$
\mathrm{SNR}_{r}=\mathrm{SNR}_{e}+10 \log _{10}\left(G_{s r}\right) \quad \text { (in decibels). }
$$

When there is no path loss, the receive SNR is equal to the equivalent $\mathrm{SNR},{ }^{4}$ which is denoted by $\mathrm{SNR}_{e}$, and $G_{s r}$ was defined in (3). Hence, a receive SNR of $-0.15 \mathrm{~dB}$ can be achieved by various combinations of $\mathrm{SNR}_{e}$ and $G_{s r}$. For the $\nu=3 \mathrm{SECCC}$ code, the decoding convergence threshold ${ }^{5}$ is at $-0.2 \mathrm{~dB}$ when employing $I=$ 40 self-concatenated iterations, which is $0.56 \mathrm{~dB}$ away from the SR link Rayleigh fading channel's capacity calculated as $-0.76 \mathrm{~dB}$ from [35] and Fig. 6 at 0.67 bit/symbol. This scheme acquires an open EXIT tunnel ${ }^{6}$ at $\mathrm{SNR}_{r}=-0.15 \mathrm{~dB}$ when communicating over an uncorrelated Rayleigh fading channel.

In our analysis, the relay node of the DSECCC-ID scheme is assumed to be placed halfway between the source and relay nodes, i.e., we have $G_{s r}=G_{r d}=4$; hence, the minimum required equivalent $\mathrm{SNR}$ at the source node is $\mathrm{SNR}_{e}=-0.15-6.02=-6.17 \mathrm{~dB}$.

Step 2: In this section, the decoding convergence of the threecomponent DSECCC-ID decoder at the destination node is analyzed. The EXIT curves of the SECCC-ID decoder at the SD link employing $I_{s d}=2$ self-concatenated iterations, as well as that of the RSC decoder recorded at the RD link, are plotted in Fig. 5. The RD link employs rates of $R_{3}=1 / 2$ and $R_{4}=1 / 2$. As shown in Fig. 5, we varied the memory of the RSC encoder to find the one that has low complexity while simultaneously matching the EXIT curve of the SECCC-ID decoder of the SD link. It can be seen from Fig. 5 that the EXIT curves associated with $\nu=2$ and $\nu=3$ do not intersect the EXIT curve of the SD link when we have $\mathrm{SNR}_{e}=-3.5 \mathrm{~dB}$ at both the SD and RD links, whereas the $\nu=4$ curve does intersect it at the same SNR. Since $\nu=2$ is a lower complexity code, we therefore opted for it for our proposed scheme.

Step 3: The convergence threshold for the DSECCC-ID system can be calculated from the EXIT curves intersecting each other at $\mathrm{SNR}_{e}$ of $-3.65 \mathrm{~dB}$. Hence, the trajectory will not reach the $(1,1)$ point of perfect convergence to a vanishingly low BER. However, once the system is operating at $\mathrm{SNR}_{e}=-3.5 \mathrm{~dB}$ at the $\mathrm{SD}$ link and again at $\mathrm{SNR}_{e}=-3.5 \mathrm{~dB}$ at the RD link, an open tunnel emerges. Since $\mathrm{SNR}_{e}=-3.5 \mathrm{~dB}$ is higher than the threshold of $\mathrm{SNR}_{e}=-6.17 \mathrm{~dB}$, which guarantees an SECCC-ID decoding convergence at the relay, the SR link may be deemed near perfect. Another reason why we configure the system to operate at a higher SNR is because we want to have fewer

\footnotetext{
${ }^{4}$ To simplify our analysis, the term "equivalent SNR" is introduced, which is the ratio of the signal power at the transmitter (source/relay node) with respect to the noise level at the receiver (relay/destination node).

${ }^{5}$ The decoding threshold is the SNR value beyond which the EXIT tunnel becomes "just" open, although this does not necessarily imply that the $\left(I_{A}, I_{E}\right)=(1,1)$ point of "perfect convergence" can be reached because some of the decoding trajectories are curtailed, owing to the limited interleaver length used.

${ }^{6}$ An open EXIT tunnel specifies the receive SNR value where there is a more widely open EXIT tunnel leading to the $(1,1)$ point and where decoding convergence to an infinitesimally low BER value can always be achieved, provided that the interleaver length is beyond a certain value, and the number of iterations is sufficiently high [19].
}

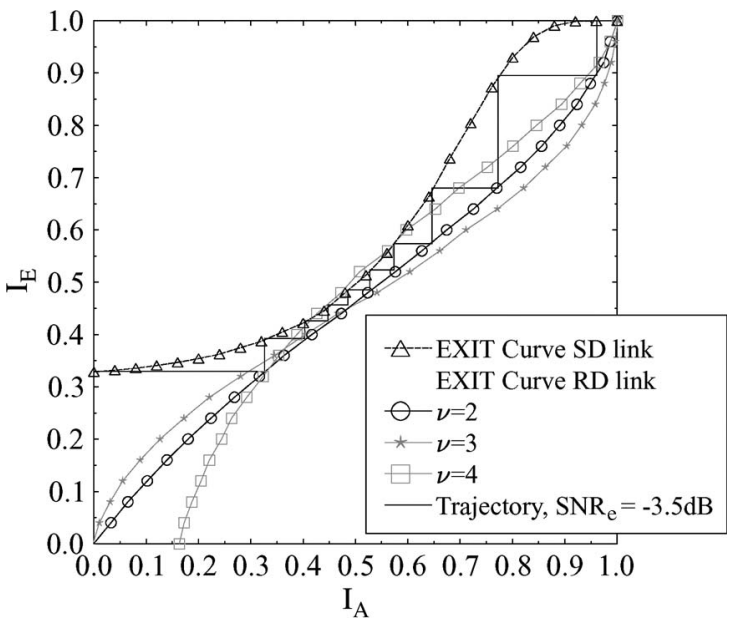

Fig. 5. EXIT curves and decoding trajectory of the DSECCC-ID scheme for $\mathrm{SNR}_{e}=-3.5 \mathrm{~dB}$ both at the source and relay nodes. The number of iterations exchanging extrinsic information between the SECCC-ID and RSC decoders at the destination node is limited to $I_{s d, r d}=10$.

self-concatenated iterations at the SR link's receiver, namely, $I_{s r}=8$ in this case.

The EXIT chart analysis is verified by computing the corresponding Monte Carlo simulation-based decoding trajectory for the DSECCCID scheme. The distinct decoding trajectory based on a frame length of 120000 bits is shown in Fig. 5 for an equivalent SNR of $-3.5 \mathrm{~dB}$ both at the source and the relay. It matches the EXIT curves generated for the SD link, which employs the SECCC-ID scheme and the RD link employing the RSC scheme, hence verifying the predicted results.

\section{B. Relay Capacity}

The two-hop half-duplex constrained relay-aided network capacity can be calculated by considering the capacity of the channel between the source, the relay, and the destination.

We first derive the upper and lower bounds on our half-duplex constrained relay-aided system's CCMC capacity and those of the DCMC capacity (constrained information rate) based on the work for full-duplex relay channels in [4]. More specifically, the upper bound of the full-duplex relay channel is given by [4]

$$
\begin{aligned}
C^{\text {Coop }} \leq \max _{p\left(x_{1}, x_{2}, x\right)} \min \{\lambda & E\left[I\left(X_{1} ; Y_{1}, Y\right)\right] \\
+ & (1-\lambda) E\left[I\left(X_{2} ; Y_{2} \mid X\right)\right], \\
& \lambda E\left[I\left(X_{1} ; Y_{1}\right)\right] \\
+ & \left.(1-\lambda) E\left[I\left(X_{2}, X ; Y_{2}\right)\right]\right\}
\end{aligned}
$$

and that of the lower bound on the CCMC and DCMC capacity of the full-duplex relay system is given in [4] as

$$
\begin{aligned}
C^{\text {Coop }} \geq \max _{p\left(x_{1}, x_{2}, x\right)} \min \{ & \lambda E\left[I\left(X_{1} ; Y\right)\right] \\
+ & (1-\lambda) E\left[I\left(X_{2} ; Y_{2} \mid X\right)\right], \\
& \lambda E\left[I\left(X_{1} ; Y_{1}\right)\right] \\
+ & \left.(1-\lambda) E\left[I\left(X_{2}, X ; Y_{2}\right)\right]\right\}
\end{aligned}
$$

where $I(A ; B)$ represents the mutual information for the channel having the i.u.d. input $A$ and the corresponding output $B$ for the case of 


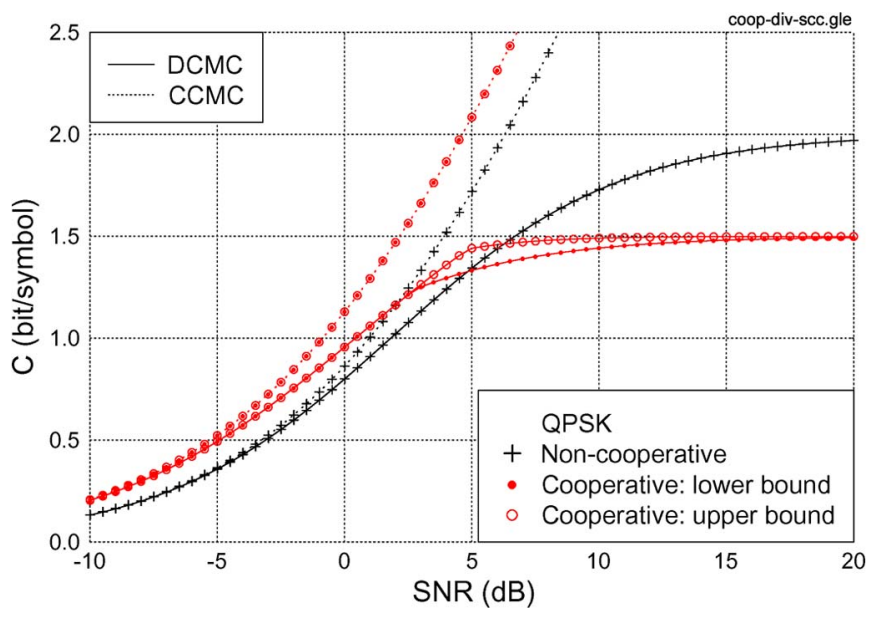

Fig. 6. DCMC and CCMC capacity curves of a cooperative network and a noncooperative QPSK-assisted scheme. It can be seen that the DCMC of a cooperative network is $-5 \mathrm{~dB}$ at $0.5 \mathrm{bit} / \mathrm{symbol}$. The DCMC of a noncooperative QPSK-assisted scheme is $-0.76 \mathrm{~dB}$ at $0.67 \mathrm{bit} / \mathrm{symbol}$.

CCMC capacity. By contrast, for the case of the DCMC, the input $A$ is constituted by phase-shift keying/quadrature-amplitude-modulated symbols. The signals $X_{1}$ and $X_{2}$ are transmitted from the source during $T_{1}$ and $T_{2}$, respectively, whereas $Y_{1}$ and $Y_{2}$ represent the corresponding signals received at the destination during the consecutive time slots. Furthermore, $X$ and $Y$ are the transmitted and received signals at the relay, respectively, and $E($.$) denotes the expectation$ with respect to the fading coefficients, $p\left(x_{1}, x_{2}, x\right)$ represents the joint probability of the signals transmitted from the source and the relay, whereas $\lambda$ is the ratio of $T_{1}$ to the total frame duration, which is given by $\left(N_{s} /\left(N_{s}+N_{r}\right)\right)=(3 / 4)$. Similarly, we have $(1-\lambda)=$ $\left(N_{r} /\left(N_{s}+N_{r}\right)\right)=(1 / 4)$.

The upper and lower bounds on the CCMC and DCMC capacity of a half-duplex, relay-aided system can then be derived by setting $X_{2}=0$, because the source does not transmit in $T_{2}$. Hence, we also have $E\left[I\left(X_{2} ; Y_{2} \mid X\right)\right]=0$ and $E\left[I\left(X_{2}, X ; Y_{2}\right)\right]=E\left[I\left(X ; Y_{2}\right)\right]$ in (13) and (14). Consequently, the upper bound can be expressed as

$$
\begin{aligned}
C^{\text {Coop }} \leq \max _{p\left(x_{1}, x\right)} \min \left\{\frac{3}{4} E\left[I\left(X_{1} ; Y_{1}, Y\right)\right]\right. & \frac{3}{4} E\left[I\left(X_{1} ; Y_{1}\right)\right] \\
& \left.+\frac{1}{4} E\left[I\left(X ; Y_{2}\right)\right]\right\}
\end{aligned}
$$

and the lower bound can be expressed as

$$
\begin{aligned}
C^{\text {Coop }} \geq \max _{p\left(x_{1}, x\right)} \min \left\{\frac{3}{4} E\left[I\left(X_{1} ; Y\right)\right], \frac{3}{4} E\right. & {\left[I\left(X_{1} ; Y_{1}\right)\right] } \\
& \left.+\frac{1}{4} E\left[I\left(X ; Y_{2}\right)\right]\right\}
\end{aligned}
$$

where the corresponding constrained information rates of $E\left[I\left(X_{1} ; Y_{1}, Y\right)\right], \quad E\left[I\left(X_{1} ; Y_{1}\right)\right], \quad E\left[I\left(X ; Y_{2}\right)\right]$, and $E\left[I\left(X_{1} ; Y\right)\right]$ can be computed by using the Monte Carlo averaging method [3]. Using (15) and (16), we can calculate the DCMC and CCMC capacity of the two-hop relay-aided network, which is graphically shown in Fig. 6.

\section{RESULTS AND Discussions}

Finally, we compare the achievable performance of the DSECCCID scheme employing a realistic relay node, which potentially induces

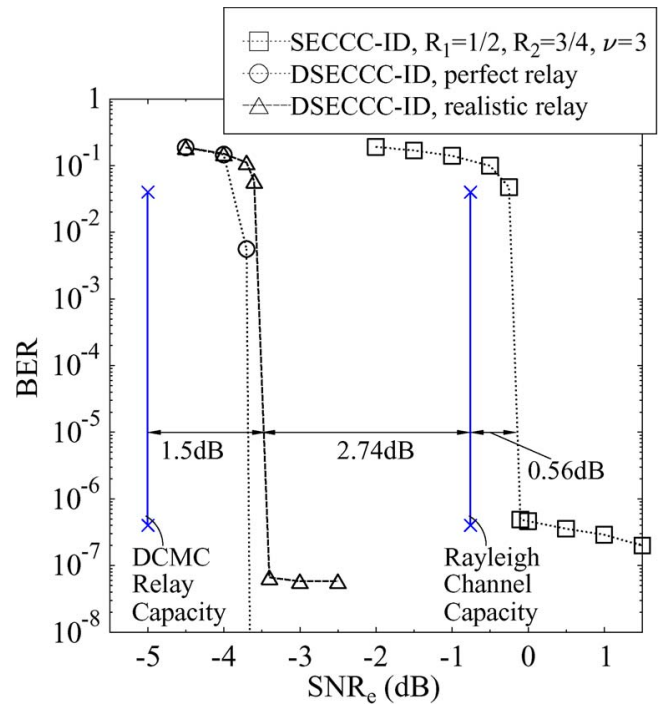

Fig. 7. BER versus equivalent SNR performance of the DSECCC-ID and SECCC-ID schemes for a frame length of 120000 bits. The DCMC relay-aided network capacity is $-5 \mathrm{~dB}$ at $0.5 \mathrm{bit} / \mathrm{symbol}$, as calculated from Fig. 6.

error propagation, with that of the noncooperative SECCC-ID scheme. The BER versus equivalent SNR performance of the DSECCC-ID and SECCC-ID schemes is shown in Fig. 7.

The SECCC-ID scheme has a decoding threshold at $-0.2 \mathrm{~dB}$ and the tunnel at $-0.15 \mathrm{~dB}$. It performs $0.56 \mathrm{~dB}$ away from the Rayleigh fading channels' capacity calculated as $-0.76 \mathrm{~dB}$ from [35] and Fig. 6 at a BER of $10^{-5}$. The DSECCC-ID system has been analyzed at $-3.5 \mathrm{~dB}$ at the source and the relay employing the RSC encoder. Thus, the DSECCC-ID scheme outperforms the SECCC-ID scheme by about $3.3 \mathrm{~dB}$ in SNR terms at a BER of $10^{-5}$, which corresponds to $3.3-1.25=2.05 \mathrm{~dB}$ in terms of $E_{b} / N_{0}$.

The performance of the DSECCC-ID scheme in conjunction with perfect relaying is shown in Fig. 7, which matches the EXIT chartbased prediction, as illustrated in Fig. 5. Furthermore, the BER performance of the DSECCC-ID scheme using perfect relaying initially matches the performance of the DSECCC-ID scheme involving realistic error-prone relaying at SNRs below the turbo cliff and rapidly improves beyond that while exhibiting no error floor, as shown in Fig. 7. The DSECCC-ID scheme assuming a realistic relay has an error floor due to errors encountered by the SECCC-ID decoder at the relay. The tunnel, as predicted by the EXIT charts shown in Fig. 5, opens at $-3.5 \mathrm{~dB}$, and beyond this point, all the trajectories reach the $(1,1)$ point. The corresponding BER curve shown in Fig. 7 matches this prediction.

As shown in Fig. 7, the proposed DSECCC-ID system is capable of performing within about $1.5 \mathrm{~dB}$ from the two-hop relay-aided network's DCMC capacity of $-5 \mathrm{~dB}$ at $0.5 \mathrm{bit} / \mathrm{symbol}$, as inferred from Fig. 6 at a BER of $10^{-5}$. In comparison, for the scheme proposed in [24], the signals arriving from the source and the relay are superimposed at the destination, where a MAP detector and a turbo decoder of memory $\nu=4$ exchange extrinsic information, which were shown to be capable of achieving a BER of $10^{-5}$, at about $1.43 \mathrm{~dB}$ away from the capacity of the ergodic flat-fading channel at an overall effective throughput of $0.44 \mathrm{bit} / \mathrm{s}$. We compare the two schemes' complexity by calculating the total number of trellis states, multiplied by the number of iterations at the corresponding decoders. This determines the number of add-compare-select (ACS) arithmetic operations of a systolic-array-based silicon chip. The total complexity of our proposed decoder is estimated as follows. 
The number of decoding iterations at the SR link's memory $\nu=3$ decoder is $I_{s r}=8$; therefore, $I_{s r} \times 2^{\nu}=8 \times 8=64$ ACS operations are required at the relay. The SD link employs $I_{s d}=2$ iterations of a memory-3 decoder, whereas the RD link employs a $\nu=2$ code. The number of iterations exchanging extrinsic information between the SECCC-ID and RSC decoders at the destination node is limited to $I_{s d, r d}=10$. Hence, the number of ACS operations at the destination is given by $I_{s d} \times 2^{3} \times 2^{2} \times I_{s d, r d}=640$. The overall ACS operations are therefore $64+640=704$.

For the case of [24], the turbo decoder at the RD link employs 15 iterations between the two parallel concatenated turbo codes, whereas 15 iterations exchange extrinsic information between the MAP decoder and the turbo decoder of memory $\nu=4$ at the destination. Hence, the overall number of ACS operations required in [24] is $(15+15) \times 2 \times 2^{4}=960$, whereas the complexity incurred by the MAP detector has not been included in the calculation. Hence, our proposed system is capable of exhibiting similar performance while incurring reduced overall complexity compared with the scheme in [24].

\section{CONCLUSION}

A power-efficient DSECCC-ID scheme has been proposed for cooperative communications. A near-capacity code, such as the SECCCID scheme, is required at the relay to minimize the decoding error probability. Once the receive SNR at the relay exceeds the error-free decoding threshold, the SECCC-ID decoder employed at the relay becomes capable of reliably decoding the source signals. The relay node employs a simple RSC encoder, and only its parity bits are transmitted to the destination. The EXIT chart of the three-component DSECCC-ID decoder reveals that the proposed system is capable of near-capacity operation at the destination, despite considering a potentially error-prone reception at the relay. The EXIT chart analysis also helps us reduce the total power required by an DSECCC-ID cooperative system by about $3.3 \mathrm{~dB}$ in SNR terms, as compared with a noncooperative SECCC-ID system at a BER of $10^{-5}$. Our future research will focus on enhancing this DSECCC-ID scheme designed for cooperative communications to operate near the capacity at low complexity using differential encoding and noncoherent detection, while dispensing with channel estimation, and will study its performance for different relay-location scenarios and power allocations. Furthermore, we will investigate the performance of such DSECCC-ID schemes in differentially encoded noncoherently detected cooperative systems.

\section{ACKNOWLEDGMENT}

The authors would like to thank the anonymous reviewers for their valuable comments, which greatly improved the presentation of this paper.

\section{REFERENCES}

[1] V. Tarokh, N. Seshadri, and A. R. Calderbank, "Space-time codes for high data rate wireless communication: Performance criterion and code construction," IEEE Trans. Inf. Theory, vol. 44, no. 2, pp. 744-765, Mar. 1998.

[2] G. Foschini, Jr. and M. Gans, "On limits of wireless communication in a fading environment when using multiple antennas," Wireless Pers. Commun., vol. 6, pp. 311-335, Mar. 1998.

[3] S. X. Ng and L. Hanzo, "On the MIMO channel capacity of multidimensional signal sets," IEEE Trans. Veh. Technol., vol. 55, no. 2, pp. 528-536, Mar. 2006.

[4] T. Cover and A. El Gamal, "Capacity theorems for the relay channel," IEEE Trans. Inf. Theory, vol. IT-25, no. 5, pp. 572-584, Sep. 1979.
[5] A. Sendonaris, E. Erkip, and B. Aazhang, "User cooperation diversity_Part I: System description," IEEE Trans. Commun., vol. 51, no. 11, pp. 1927-1938, Nov. 2003.

[6] J. N. Laneman, D. N. C. Tse, and G. W. Wornell, "Cooperative diversity in wireless networks: Efficient protocols and outage behavior," IEEE Trans. Inf. Theory, vol. 50, no. 12, pp. 3062-3080, Dec. 2004.

[7] E. Zimmermann, P. Herhold, and G. Fettweis, "On the performance of cooperative relaying protocols in wireless networks," Eur. Trans. Telecommun., vol. 16, no. 1, pp. 5-16, Jan./Feb. 2005.

[8] H. Ochiai, P. Mitran, and V. Tarokh, "Design and analysis of collaborative diversity protocols for wireless sensor networks," in Proc. IEEE VTC-Fall, Los Angeles, CA, Sep. 26-29, 2004, pp. 4645-4649.

[9] J. Abouei, H. Bagheri, and A. Khandani, "An efficient adaptive distributed space-time coding scheme for cooperative relaying," IEEE Trans. Wireless Commun., vol. 8, no. 10, pp. 4957-4962, Oct. 2009.

[10] A. Host-Madsen and J. Zhang, "Capacity bounds and power allocation for wireless relay channels," IEEE Trans. Inf. Theory, vol. 51, no. 6, pp. 2020-2040, Jun. 2005.

[11] S. X. Ng, J. Wang, and L. Hanzo, "Unveiling near-capacity code design: The realization of Shannon's communication theory for MIMO channels," in Proc. IEEE ICC, May 2008, pp. 1415-1419.

[12] G. Forney, Concatenated Codes. Cambridge, MA: MIT Press, 1966.

[13] C. Berrou, A. Glavieux, and P. Thitimajshima, "Near Shannon limit error correcting coding and decoding: Turbo codes," in Proc. IEEE ICC, 1993, pp. 1064-1070.

[14] S. Le Goff, A. Glavieux, and C. Berrou, "Turbo-codes and high spectral efficiency modulation," in Proc. IEEE Int. Conf. Commun., New Orleans, LA, May 1994, pp. 645-649.

[15] S. Benedetto, D. Divsalar, G. Montorsi, and F. Pollara, "Bandwidth efficient parallel concatenated coding schemes," Electron. Lett., vol. 31, no. 24, pp. 2067-2069, Nov. 1995.

[16] P. Robertson and T. Worz, "Coded modulation scheme employing turbo codes," Electron. Lett., vol. 31, no. 18, pp. 1546-1547, Aug. 1995.

[17] S. Benedetto, D. Divsalar, G. Montorsi, and F. Pollara, "Serial concatenated trellis coded modulation with iterative decoding," in Proc. IEEE Int. Symp. Inf. Theory, Ulm, Germany, Jun./Jul. 1997, p. 8.

[18] S. Benedetto, D. Divsalar, G. Montorsi, and F. Pollara, "Self-concatenated trellis coded modulation with self-iterative decoding," in Proc. IEEE Global Telecommun. Conf., Sydney, Australia, 1998, vol. 1, pp. 585-591.

[19] S. ten Brink, "Convergence behavior of iteratively decoded parallel concatenated codes," IEEE Trans. Commun., vol. 49, no. 10, pp. 1727-1737, Oct. 2001.

[20] M. F. U. Butt, R. A. Riaz, S. X. Ng, and L. Hanzo, "Near-capacity iteratively decoded binary self-concatenated code design using EXIT charts," in Proc. IEEE GLOBECOM, New Orleans, LA, Nov./Dec. 2008, pp. $1-5$.

[21] Y. Li, "Distributed coding for cooperative wireless networks: An overview and recent advances," IEEE Commun. Mag., vol. 47, no. 8, pp. 71-77, Aug. 2009.

[22] B. Zhao and M. C. Valenti, "Distributed turbo coded diversity for relay channel," Electron. Lett., vol. 39, no. 10, pp. 786-787, May 2003.

[23] M. Janani, A. Hedayat, T. E. Hunter, and A. Nosratinia, "Coded cooperation in wireless communications: Space-time transmission and iterative decoding," IEEE Trans. Signal Process., vol. 52, no. 2, pp. 362-371, Feb. 2004.

[24] Z. Zhang and T. M. Duman, "Capacity-approaching turbo coding for halfduplex relaying," IEEE Trans. Commun., vol. 55, no. 10, pp. 1895-1906, Oct. 2007.

[25] J. Hu and T. M. Duman, "Low density parity check codes over wireless relay channels," IEEE Trans. Wireless Commun., vol. 6, no. 9, pp. 33843394, Sep. 2007.

[26] L. Hanzo, O. Alamri, M. El-Hajjar, and N. Wu, Near-Capacity Multi-Functional MIMO Systems. Hoboken, NJ: Wiley-IEEE Press, Apr. 2009.

[27] M. Elfituri, W. Hamouda, and A. Ghrayeb, "A convolutional-based distributed coded cooperation scheme for relay channels," IEEE Trans. Veh. Technol., vol. 58, no. 2, pp. 655-669, Feb. 2009.

[28] H. H. Sneesens and L. Vandendorpe, "Soft decode and forward improves cooperative communications," in Proc. 6th IEE Int. Conf. 3 G Beyond, Washington, DC, Nov. 2005, pp. 1-4.

[29] Y. Li, B. Vucetic, T. F. Wong, and M. Dohler, "Distributed turbo coding with soft information relaying in multihop relay networks," IEEE J. Sel. Areas Commun., vol. 24, no. 11, pp. 2040-2050, Nov. 2006.

[30] R. U. Nabar, H. Bolcskei, and F. W. Kneubuhler, "Fading relay channels: Performance limits and space-time signal design," IEEE J. Sel. Areas Commun., vol. 22, no. 6, pp. 1099-1109, Aug. 2004. 
[31] M. C. Valenti and B. Zhao, "Distributed turbo codes: Towards the capacity of the relay channel," in Proc. IEEE 58th VTC-Fall, Oct. 2003, vol. 1, pp. 322-326.

[32] S. X. Ng, M. F. U. Butt, and L. Hanzo, "On the union bounds of selfconcatenated convolutional codes," IEEE Signal Process. Lett., vol. 16, no. 9, pp. 754-757, Sep. 2009.

[33] J. Hagenauer, "Rate-compatible punctured convolutional codes (RCPC codes) and their applications," IEEE Trans. Commun., vol. 36, no. 4, pp. 389-400, Apr. 1988.

[34] S. Benedetto, D. Divsalar, G. Motorsi, and F. Pollara, "A soft-input softoutput APP module for iterative decoding of concatenated codes," IEEE Commun. Lett., vol. 1, no. 1, pp. 22-24, Jan. 1997.

[35] L. Hanzo, S. X. Ng, T. Keller, and W. Webb, Quadrature Amplitude Modulation: From Basics to Adaptive Trellis-Coded, Turbo-Equalised and Space-Time Coded OFDM, CDMA and MC-CDMA Systems, 2nd ed. Hoboken, NJ: Wiley-IEEE Press, Dec. 15, 2004, pp. 746-748.

\section{A Novel Interference-Avoidance Code Reassignment for Downlink Two-Dimensional-Spread MC-DS-CDMA Systems With Power Control}

\author{
Chih-Wen Chang and Chien-Cheng Kuo
}

\begin{abstract}
In the 2-D time- and frequency-domain spread multicarrier direct-sequenced code-division multiple-access (MC-DS-CDMA) system, maintaining a good code tree structure is the key to controlling the interference level. However, as users come and go, the code tree structure will be fragmented and steeped in a high level of interference. In this situation, an active call may be dropped for not being able to reach a predefined signal quality. To solve this call-dropping problem, we propose a novel code-reassignment strategy to reallocate a low-interferenced code to the could-be-dropped call. This way, not only can this possible call-dropping event be evaded, but also, a low-interferenced 2-D code tree structure can be maintained. The simulation results show that the proposed codereassignment method can efficiently alleviate the call-dropping problem and boost the call-survival rate simultaneously. It is believed that the proposed scheme fills up an important missing link of the dynamic callresource management for the 2-D-spread MC-DS-CDMA system.
\end{abstract}

Index Terms-Code assignment and reassignment, interference avoidance, multiple-access interference (MAI) coefficient, multicarrier direct-sequenced code-division multiple access (MC-DS-CDMA), power control (PC).

\section{INTRODUCTION}

In recent years, the family of the multicarrier code-division multiple-access (MC-CDMA) transmission technologies have drawn a lot of attention for its ability and flexibility to provide ubiquitous broadband wireless communications [1], [2]. Among the MC-CDMA family, the 2-D spread version of multicarrier direct-sequenced CDMA (MC-DS-CDMA) can enhance the system capacity by sharing distinct codes in either time or frequency domains. However, the nonorthogonality of the frequency-domain spreading codes in the frequencyselective fading channel can lead to the undesirable multiple-access interference (MAI), and consequently, the system performance can

Manuscript received September 29, 2009; revised January 31, 2010; accepted Macrh 6, 2010. Date of publication April 5, 2010; date of current version July 16, 2010. This work was supported in part by the National Science Council, Taiwan, under Contracts 96-2221-E-006-020 and 97-2221-E-006-085-MY3. The review of this paper was coordinated by Prof. H. Liu.

The authors are with the Institute of Computer and Communication Engineering, National Cheng Kung University, Tainan 701, Taiwan (e-mail: cwchang@ee.ncku.edu.tw).

Digital Object Identifier 10.1109/TVT.2010.2047329 seriously be degraded. Thus, how to effectively eliminate the MAI in the 2-D-spread MC-DS-CDMA system becomes a critical issue. In the literature, several aspects have been explored to eliminate this kind of MAI for the 2-D-spread MC-DS-CDMA, including the sophisticated multiuser detections [3], interference rejection time-domain spreading code for the quasi-synchronous transmissions [4], joint beamforming and steered space-time spreading schemes [5], and interference avoidance (IA) code assignment strategies [6]-[8]. In this paper, we propose a code-reassignment strategy to further enhance the capability of the joint power control (PC) and IA code-assignment (denoted by $\mathrm{PC}+\mathrm{IA}$ ) strategy in [8] for eliminating the MAI.

In the conventional 1-D spread downlink CDMA system, the main principle of accepting a call request is the availability of the spreading code resources. Therefore, some code-assignment and reassignment strategies have been proposed in [9] and [10] to solve the call-blocking problem by improving the trunking efficiency of the code resources. In addition, several quality-based code-assignment and reassignment schemes have been proposed to efficiently adapt the data-transmission rates to the link qualities [11]. Differently, in the 2-D-spread MC-DSCDMA system, the key of accommodating a coming call request lies in the MAI level. In addition, maintaining a good code-tree structure is critical to control the level of MAI. Therefore, to keep the call-tree structure in good shape, the IA code-assignment strategies associated with the subcarrier power allocation and the PC mechanism have been proposed in [6]-[8], respectively. However, as users come and go, the code-tree structure will be fragmented and steeped in a high level of MAI. In this situation, an active call may confront the call-dropping problem owing to the unsatisfactory signal quality for a consecutive period of time.

To solve this kind of call-dropping problem, on top of the previous $\mathrm{PC}+\mathrm{IA}$ code-assignment scheme, we propose a novel codereassignment strategy to keep a low-interferenced 2-D code-tree structure. The proposed code-reassignment procedure will be launched when the predefined signal-to-interference-and-noise ratio (SINR) of an active call cannot satisfy the target value for a consecutive period of time. Then, based on an MAI coefficient evaluation, this active call will be reassigned a less interfered code. Accordingly, a possible call-dropping event can be avoided if the SINR associated with the reassigned code can reach the target value. The simulation results show that the proposed code-reassignment scheme can efficiently alleviate the call-dropping problem and boost the call survival rate simultaneously. To summarize, one can say that employing the codereassignment scheme to enhance the $\mathrm{PC}+\mathrm{IA}$ code-assignment strategy is imperative to achieve the dynamic code-resource management for the 2-D-spread MC-DS-CDMA system.

The rest of this paper is organized as follows: In Section II, we introduce the background knowledge of the 2-D-spread MC-DS-CDMA system and address the motivation of this paper. Section III proposes the novel IA code-reassignment strategy. The simulation results are provided in Section IV. Section V gives a concluding remark and some discussions.

\section{BACKGROUND, MOTIVATION, AND CONCEPT OF CODE REASSIGNMENT}

\section{A. Grid Representation of the 2-D OVSF Code Tree}

Following the same system model and assumptions of [7], [8], and [12], in the downlink 2-D-spread MC-DS-CDMA systems, the orthogonal variable spreading factor (OVSF) code tree has a 2-D structure. A so-called grid representation was introduced in [6] to illustrate the code resources using a set of rectangles. Code channels with the same 